\title{
Efficacy of Fungicide Treatments for Control of Common Rust and Northern Leaf Spot in Hybrid Corn Seed Production
}

\author{
S. N. Wegulo, J. M. Rivera-C, C. A. Martinson, and F. W. Nutter, Jr., Department of Plant Pathology, Iowa State \\ University, Ames 50011
}

\begin{abstract}
Wegulo, S. N., Rivera-C, J. M., Martinson, C. A., and Nutter, F. W., Jr. 1998. Efficacy of fungicide treatments for control of common rust and northern leaf spot in hybrid corn seed production. Plant Dis. 82:547-554.

Field experiments were conducted during the 1992 and 1993 growing seasons in 13 commercial fields in Iowa to assess the efficacy of foliar applications of fungicides to seed corn for control of common rust, Puccinia sorghi, and northern leaf spot, Bipolaris zeicola. Chlorothalonil, mancozeb, and/or propiconazole sprays were initiated at growth stage (GS) 2.5, GS 4.0, or approximately 2 weeks before detasseling, and applications were repeated up to five times at various growth stages. The majority of fungicide treatments resulted in an increase in salable seed units ( 1 unit $=80,000$ seeds) compared to untreated control treatments. The greatest increase in salable seed occurred in the large and medium seed size units. Although disease severity on the final assessment date in both years and standardized area under the disease progress curve in 1993 were significantly higher $(P \leq 0.05)$ in untreated control plots than in fungicide-treated plots in all experiments, the best disease control was provided by 5 applications of chlorothalonil or 2 to 4 applications of chlorothalonil, mancozeb, or propiconazole initiated on the first spray date.
\end{abstract}

Additional keywords: Aureobasidium zeae, Cercospora zeae-maydis, Exserohilum turcicum

Common leaf rust of corn, caused by Puccinia sorghi, is prevalent in the Midwestern Unites States. Epidemic outbreaks of the disease on corn inbreds occur frequently when environmental conditions during the growing season are favorable. Few studies $(13,23)$ have been conducted to determine the effects of foliar applications of fungicides on common rust severity and yield in corn inbreds. Investigations concerning the relationship between common rust severity and yield loss in corn and the efficacy of fungicides in controlling the disease have been conducted mostly on hybrid sweet corn $(3,8,18-21)$. These studies have demonstrated the effectiveness of fungicides in controlling common rust and in reducing yield loss. Pataky and Eastburn (19) found that the level of common rust control

Corresponding author: C. A. Martinson E-mail: CMARTINS@IASTATE.EDU

Journal Paper No. J-16921 of the Iowa Agriculture and Home Economics Experiment Station, Projects No. 2646 and 3394. Portions of a Ph.D. dissertation by J. M. Rivera-C and an M. S. thesis by S. N. Wegulo submitted to the Graduate College, Iowa State University. This research was funded in part by USDA-CSRS North Central Region Pesticide Impact Assessment Grant No. 553.

Accepted for publication 2 February 1998.

Publication no. D-1998-0305-02R

(C) 1998 The American Phytopathological Society provided by partial resistance in hybrid sweet corn depended, in part, on the effectiveness of fungicides. Five applications of mancozeb or propiconazole reduced common rust by about $55 \%$ on a susceptible sweet corn hybrid and by about $40 \%$ on partially resistant and resistant hybrids compared to no fungicidal control on the same hybrids.

Northern leaf spot, caused by Bipolaris zeicola, is one of the more prevalent pathogens of corn across the Midwestern United States and is particularly damaging where seed corn follows corn, especially when highly susceptible inbreds are grown (4). Fisher et al. (5) and Ullstrup and Miles (28) have reported yield losses caused by northern leaf spot of up to $80 \%$ in corn. Although the severity of the disease can be reduced by use of foliar fungicides, the management practices mostly relied upon by producers are host resistance, conventional tillage practices, and crop rotation (25).

Because inbred lines are homozygous, the reaction to diseases is likely to be more disperse among a large group of inbreds compared to a large group of hybrids. Some popular inbred lines may be more susceptible to common rust than most hybrids. When such inbred lines are used in hybrid corn seed production, there is great potential for yield reduction when conditions are favorable for common rust epidemics. Hence, there is a need for quantitative information concerning the re- lationship between levels of common rust severity and seed yield in hybrid corn seed production. Quantitative information is also needed concerning the efficacy of fungicides in controlling common rust in hybrid corn seed production fields.

Hybrid corn seed is a high-value commodity, mainly because of the extra effort required to prevent self-pollination of inbreds. Prevention of self-pollination is necessary to maximize genetic purity of hybrid seed, and it is prevented by detasseling, the physical removal of the tassel from the seed parent plant before pollen shed or silking. Detasseling can be achieved manually by hand or in combination with machines. Because of insufficient labor supply and the high cost of handdetasseling ( $\$ 250$ to $\$ 320$ per seed parent hectare; 35), machines have increasingly been used to detassel seed parent inbreds. Mechanical detasseling removes a significant amount of photosynthetically active leaf area, which reduces seed yields $(1,10,29,35)$. It follows that additional reductions in photosynthetic leaf area caused by foliar diseases may have a significant negative effect on seed yield. Although the imminent registration of a chemical compound that induces male sterility in seed parent inbred corn plants when applied approximately 4 weeks before flowering may somewhat reduce this increased negative effect of mechanical detasseling on yield (15), the planting of susceptible inbreds coupled with environmental conditions that favor disease development may still require the use of fungicides to prevent reductions in seed yields.

In addition, emphasis in recent years on minimum- or no-tillage practices to reduce soil erosion (27) has led to increased survival of several foliar pathogens during intercrop periods, including Aureobasidium zeae, Bipolaris zeicola, Cercospora zeaemaydis, and Exserohilum turcicum. The occurrence of diseases incited by these pathogens in addition to common rust during the same growing season may result in increased yield reductions. Therefore, it may be necessary to use fungicides to reduce the risk of yield loss caused by common rust and other foliar fungal diseases associated with hybrid corn seed production. Economic benefits of using fungicides to control foliar fungal diseases of corn inbreds have been demonstrated $(33,34)$. The purpose of this study was to evaluate the efficacy of chlorothalonil, 
mancozeb, and propiconazole in controlling common rust and northern leaf spot in corn inbreds and in increasing units of salable hybrid corn seed.

\section{MATERIALS AND METHODS}

Field trials. Experiments were conducted in Iowa in commercial corn seed production fields in 1992 and 1993 on land planted to corn the previous production year. Infested corn debris provided the initial inoculum for diseases incited by $A$. zeae, B. zeicola, C. zeae-maydis, and $E$. turcicum. Preliminary experiments were conducted in 1990 (Henry, Jefferson, Mahaska, and Marshall Counties) and 1991 (Cedar and Mahaska Counties). In 1992, experiments were conducted in Benton, Grundy, Marshall, and Tama Counties; and in 1993, experiments were conducted in Benton, Grundy, Marshall, Story, and Webster Counties. The seed parent inbreds planted in each experiment, including preliminary experiments, were numbered arbitrarily from 1 to 7 . Inbreds 1 and 3 were planted in 1992 and inbreds 4 to 7 were planted in 1993. In 1992, the majority of our experiments were conducted in fields planted by one seed company; therefore, it was possible to replicate by location experiments in which inbred 1 was planted. In 1993, a different inbred was planted in each of four geographical areas, and there were two replicates of each experiment in each geographical area. The two replicated experiments in each area were separated by a distance of 1 to $8 \mathrm{~km}$.

Experimental units were plots of seed parent plants $11 \mathrm{~m}$ long and 4 rows wide. Row spacing was $0.76 \mathrm{~m}$. The 4 rows of seed parent plants in each plot alternated with 1 row of pollen parent inbred plants. Treatments were arranged in a randomized complete block design with four replications. In treatments with multiple applications, one spray was applied at each growth stage. Application rates were 1.69, 1.26 , and $0.12 \mathrm{~kg}$ a.i./ha for chlorothalonil, mancozeb, and propiconazole, respectively. Latron-B 1956, a spreader-sticker, was added to mancozeb at 0.15 liter/ha at each site. Fungicide application equipment consisted of a 4-row spray boom with 3 TXVS-3 Conejet nozzles (Spraying Systems Co., Wheaton, IL) per row. A motorized backpack sprayer (Model SHR-200E, ECHO Inc., Northbrook, IL) powered the boom. One nozzle was positioned approximately $0.15 \mathrm{~m}$ above each row, and 2 nozzles were each attached to $0.38-\mathrm{m}$ hose drops (Spraying Systems Co., Wheaton, IL) positioned midway between rows. The boom extended over 6 rows (the 4 sprayed seed parent rows and 1 unsprayed pollen parent row on each side) and was carried by 2 persons. Each person held one end of the spray boom and walked at a timed pace of $0.44 \mathrm{~m} / \mathrm{s}$ to deliver approximately 341 liters/ha of fungicide spray at $414 \mathrm{kPa}$.

In 1992, fungicide sprays were timed to coincide with specific growth stages ranging from growth stage (GS) 2.5 to GS 7.0 (Table 1; 9). In fields $92 \mathrm{~A}$ to $\mathrm{D}$, chlorothalonil treatments consisted of sprays applied at (i) GS 2.5, 4.0, 5.5, and 7.0; (ii) GS 2.5 and 4.0; (iii) GS 4.0 and 5.5; (iv) GS 5.5 and 7.0; (v) GS 4.0; and (vi) GS 5.5. A single mancozeb treatment consisted of sprays applied at GS 2.5, 4.0, 5.5, and 7.0. Propiconazole sprays were applied at (i) GS 2.5 and 4.0, and (ii) GS 4.0.

In field $92 \mathrm{E}$, chlorothalonil sprays were applied at (i) GS 4.0, 5.5, and 7.0; (ii) GS 4.0 and 5.5; (iii) GS 5.5 and 7.0; (iv) GS 4.0, and (v) GS 5.5. There was one mancozeb treatment which consisted of sprays applied at GS 4.0, 5.5, and 7.0. The first propiconazole treatment in this field consisted of sprays at GS 4.0 and 5.5; the second consisted of one spray at GS 4.0. In one treatment, one propiconazole spray was applied at GS 4.0 and was followed by one chlorothalonil spray at GS 5.5.

In 1993, the first, second, and third sprays were applied approximately 7 to 14 days before detasseling, 1 to 4 days before the first day of detasseling, and 1 to 4 days after the last day of detasseling. The fourth and fifth sprays were applied at approxi-

Table 1. Descriptions of growth stages of corn and their identifying characteristics used in fungicide experiments in hybrid corn seed production fields in Iowa in $1992^{\mathrm{z}}$

\begin{tabular}{l}
\hline Growth stage (GS) \\
\hline Vegetative stages \\
GS 0.0 \\
GS 1.0 \\
GS 2.0 \\
GS 3.0 \\
GS 4.0 \\
Reproductive stages \\
GS 5.0 \\
GS 6.0 \\
GS 7.0 \\
GS 8.0 \\
GS 9.0 \\
GS 10.0
\end{tabular}

Identifying characteristics

Plant emergence. Tip of coleoptile visible at soil surface. Collar of 4th leaf visible. Collar of 8th leaf visible. Collar of 12th leaf visible. Collar of 16th leaf visible.

$50 \%$ of plants have silks visible. Ears have most kernels in "blister" stage. Ears have most kernels in "soft dough" stage. In some ears kernels start denting. Kernels fully dented.

Black layer formation. Physiological maturity reached, dry matter accumulation ceased.

${ }^{\mathrm{z}}$ Modified from Hanway (9). For the identification of transition phases between stages, the interval was arbitrarily divided in three substages equally spaced in time, identified as $0.2,0.5$, and 0.8 . mately 10-day intervals after the third spray. Five chlorothalonil treatments were applied and consisted of sprays on (i) the first, second, third, fourth, and fifth application dates, (ii) the first and second application dates, (iii) the second and third application dates, (iv) the second application date, and (v) the second, third, and fourth application dates. One mancozeb treatment consisted of sprays applied on the first, second, and third application dates. Two propiconazole treatments consisted of sprays applied on (i) the first and second application dates, and (ii) the second application date. In one treatment, two propiconazole sprays were applied on the first and second application dates and were followed by one mancozeb spray on the third application date.

To assess disease, 5 or 6 seed parent inbred plants were arbitrarily selected from the middle two rows of each plot, excluding plants within $0.5 \mathrm{~m}$ at either end of each row. In 1992, disease severity was assessed at GS 8.2 in field 92 A, GS 7.0 and 8.5 in fields 92 B to D, and GS 8.5 and 9.5 in field 92 E. In 1993, a preliminary disease severity assessment of the overall experimental area was made on the date the first fungicide spray was applied. Thereafter, disease severity was assessed at approximately 7- to 14-day intervals starting at 1 to 7 days after the last date of detasseling. Percentage of leaf area covered with lesions was estimated with the aid of standard area diagrams (11) on the ear immediately above the ear leaf, the ear leaf, and the two leaves immediately below the ear leaf. Disease data were fit to disease progress models using a computer program (17). Critical point models $(2,16)$ were used to determine assessment dates on which disease severity levels had the best relationship to yield. In 1992, there was an insufficient number of disease severity assessment dates ( 1 to 3 ) to calculate area under disease progress curve (AUDPC) values. In 1993, there were six disease severity assessments in each field and AUDPC was approximated by trapezoidal integration (24). Standardized (relative) AUDPC values were obtained by dividing AUDPC values by the total time duration (days) of each epidemic (6).

At maturity, ears from the middle $10 \mathrm{~m}$ of seed parent rows in each plot were hand-harvested and immediately delivered to the respective cooperating seed companies (Pioneer Hi-Bred International Inc., ICI Seeds Inc., and Lynks Seeds Inc.) for drying, shelling, weighing, and sizing. Seed samples weighing $0.5 \mathrm{~kg}$ were sized by the seed companies or at Iowa State University into the following categories: (i) large size (passed through a $0.95-\mathrm{cm}$ diameter [24/64 in.-hole] screen and retained on a $0.87-\mathrm{cm}$ diameter [22/64 in.hole] screen), (ii) medium size (passed through a $0.87-\mathrm{cm}$ diameter screen and retained on a $0.79-\mathrm{cm}$ diameter [20/64 in.- 
hole] screen), (iii) small size (passed through a $0.79-\mathrm{cm}$ diameter screen and retained on a $0.64-\mathrm{cm}$ diameter [16/64 in.hole] screen), and (iv) rejected seed (retained on a $0.95-\mathrm{cm}$ diameter screen or passed through a $0.64-\mathrm{cm}$ diameter screen). The sized fractions (large, medium, and small) obtained from the yield harvested from each plot were weighed, and from these fractions 200 seeds were selected randomly and weighed. All seeds in a fraction were weighed if there were fewer than
200. Weight per kernel for each fraction was determined by dividing the weight of the sample by the number of seeds in the sample. These data were used to calculate the number of units of salable seed $(80,000$ seeds/unit at $12 \%$ moisture) per hectare.

Data analysis. Data were analyzed by the general linear models procedure. The ratio test and Bartlett's test of homogeneity of variance (22) were used to determine whether or not to perform combined analyses of disease severity and yield data from two or more experiments in which the same seed parent and pollen parent inbreds were planted. The least significant difference test (26) was used to make planned pairwise means comparisons for disease estimates and units of salable seed. Linear regression analysis was used to quantify the relationships between disease severity assessments, standardized AUDPC, and seed corn yield (total units of salable seed). Coefficients of determination $\left(R^{2}\right), F$ tests, and standard errors of the $Y$-estimate

Table 2. Large, medium, small, and total salable seed units, and disease severity from fungicide treatments in experimental plots in hybrid corn seed production fields in Iowa in 1992

\begin{tabular}{|c|c|c|c|c|c|c|c|c|}
\hline Treatment $^{w}$ & $\begin{array}{c}\text { Large } \\
\text { units/ha }\end{array}$ & $\begin{array}{c}\text { Medium } \\
\text { units/ha }\end{array}$ & $\begin{array}{c}\text { Small } \\
\text { units/ha }\end{array}$ & $\begin{array}{c}\text { Total } \\
\text { units/ha }\end{array}$ & $\begin{array}{c}\% \text { LAD }^{x} \\
\text { at GS 7.0 }\end{array}$ & $\begin{array}{c}\text { \%LAD } \\
\text { at GS } 8.2\end{array}$ & $\begin{array}{c}\text { \%LAD } \\
\text { at GS } 8.5\end{array}$ & $\begin{array}{c}\text { \%LAD } \\
\text { at GS } 9.5\end{array}$ \\
\hline \multicolumn{9}{|c|}{$\begin{array}{l}\text { Field } 92 \text { A, Benton County, } \\
\text { Inbred } 1\end{array}$} \\
\hline Mancozeb $1,2,3,4^{y}$ & $9.08 \mathrm{a}$ & $66.0 \mathrm{a}$ & 143.8 & 218.9 & $\ldots$ & $0.6 \mathrm{c}$ & $\ldots$ & $\ldots$ \\
\hline Chlorothalonil 1,2,3,4 & $4.88 \mathrm{~b}$ & $54.9 \mathrm{~b}$ & 157.7 & 217.4 & $\ldots$ & $2.0 \mathrm{c}$ & $\ldots$ & $\ldots$ \\
\hline Chlorothalonil 1,2 & $4.40 \mathrm{~b}$ & $54.0 \mathrm{~b}$ & 149.5 & 207.9 & $\ldots$ & $5.3 \mathrm{~b}$ & $\ldots$ & $\ldots$ \\
\hline Chlorothalonil 2,3 & $4.76 \mathrm{~b}$ & $51.3 \mathrm{~b}$ & 154.5 & 210.6 & $\ldots$ & $5.1 \mathrm{~b}$ & $\ldots$ & $\ldots$ \\
\hline Chlorothalonil 2 & $5.49 \mathrm{~b}$ & $56.7 \mathrm{~b}$ & 151.9 & 214.0 & $\ldots$ & $5.2 \mathrm{~b}$ & $\ldots$ & $\ldots$ \\
\hline Chlorothalonil 3,4 & $3.54 \mathrm{~b}$ & $50.3 \mathrm{~b}$ & 153.6 & 207.4 & $\ldots$ & $6.7 \mathrm{~b}$ & $\ldots$ & $\ldots$ \\
\hline Chlorothalonil 3 & $4.91 \mathrm{~b}$ & $52.0 \mathrm{~b}$ & 156.0 & 212.9 & $\ldots$ & $6.7 \mathrm{~b}$ & $\ldots$ & $\ldots$ \\
\hline Propiconazole 1,2 & $3.95 \mathrm{~b}$ & $55.4 \mathrm{~b}$ & 146.5 & 205.9 & $\ldots$ & $6.6 \mathrm{~b}$ & $\ldots$ & $\ldots$ \\
\hline Propiconazole 2 & $3.52 \mathrm{~b}$ & $51.7 \mathrm{~b}$ & 155.9 & 211.1 & $\ldots$ & $7.0 \mathrm{~b}$ & $\ldots$ & $\ldots$ \\
\hline Untreated & $4.26 \mathrm{~b}$ & $50.8 \mathrm{~b}$ & 149.1 & 204.2 & $\ldots$ & $9.7 \mathrm{a}$ & $\ldots$ & $\ldots$ \\
\hline $\mathrm{CV}(\%)^{\mathrm{z}}$ & 36.1 & 11.3 & 6.1 & 4.2 & $\ldots$ & 24.9 & $\ldots$ & $\ldots$ \\
\hline \multicolumn{9}{|c|}{$\begin{array}{l}\text { Field } 92 \text { B, Benton County, } \\
\text { Inbred } 1\end{array}$} \\
\hline Mancozeb 1,2,3,4 & $2.14 \mathrm{ab}$ & $45.2 \mathrm{a}$ & 173.5 & 220.9 a & $0.8 \mathrm{~d}$ & $\ldots$ & $0.9 \mathrm{~b}$ & $\ldots$ \\
\hline Chlorothalonil 1,2,3,4 & $2.41 \mathrm{a}$ & $41.9 \mathrm{a}$ & 160.7 & $205.1 \mathrm{a}-\mathrm{c}$ & $2.2 \mathrm{bc}$ & $\ldots$ & $2.8 \mathrm{a}$ & $\ldots$ \\
\hline Chlorothalonil 1,2 & $0.90 \mathrm{c}-\mathrm{e}$ & $25.6 \mathrm{de}$ & 172.0 & $198.5 \mathrm{a}-\mathrm{d}$ & $2.1 \mathrm{c}$ & $\ldots$ & $3.1 \mathrm{a}$ & $\ldots$ \\
\hline Chlorothalonil 2,3 & $0.97 \mathrm{c}-\mathrm{e}$ & $31.5 \mathrm{bc}$ & 176.0 & $208.5 \mathrm{a}-\mathrm{c}$ & $4.1 \mathrm{a}$ & $\ldots$ & $3.8 \mathrm{a}$ & $\ldots$ \\
\hline Chlorothalonil 2 & $0.28 \mathrm{e}$ & $20.5 \mathrm{ef}$ & 164.2 & $184.9 \mathrm{~cd}$ & $2.5 \mathrm{bc}$ & $\ldots$ & $3.8 \mathrm{a}$ & $\ldots$ \\
\hline Chlorothalonil 3,4 & $1.79 \mathrm{a}-\mathrm{c}$ & $34.3 \mathrm{~b}$ & 167.0 & $203.1 \mathrm{a}-\mathrm{d}$ & $4.2 \mathrm{a}$ & $\ldots$ & $4.1 \mathrm{a}$ & $\ldots$ \\
\hline Chlorothalonil 3 & $1.35 \mathrm{~b}-\mathrm{d}$ & $29.4 \mathrm{~b}-\mathrm{d}$ & 160.0 & $190.7 \mathrm{~b}-\mathrm{d}$ & $3.5 \mathrm{ab}$ & $\ldots$ & $3.0 \mathrm{a}$ & $\ldots$ \\
\hline Propiconazole 1,2 & $0.62 \mathrm{de}$ & $24.3 \mathrm{de}$ & 160.8 & $185.7 \mathrm{~cd}$ & $2.5 \mathrm{bc}$ & $\ldots$ & $3.9 \mathrm{a}$ & $\ldots$ \\
\hline Propiconazole 2 & $0.44 \mathrm{de}$ & $26.5 \mathrm{~cd}$ & 184.1 & $211.1 \mathrm{ab}$ & $2.5 \mathrm{bc}$ & $\ldots$ & $3.4 \mathrm{a}$ & $\ldots$ \\
\hline Untreated & $0.34 \mathrm{e}$ & $17.8 \mathrm{f}$ & 161.0 & $179.1 \mathrm{~d}$ & $2.4 \mathrm{bc}$ & $\ldots$ & $3.4 \mathrm{a}$ & $\ldots$ \\
\hline $\mathrm{CV}(\%)$ & 59.3 & 12.5 & 9.6 & 8.8 & 33.9 & $\ldots$ & 29.2 & $\ldots$ \\
\hline \multicolumn{9}{|c|}{ Fields $92 \mathrm{C}$ and $\mathrm{D}$, Marshall and } \\
\hline \multicolumn{9}{|c|}{ Grundy Counties, Inbred 1} \\
\hline Mancozeb 1,2,3,4 & $13.04 \mathrm{a}$ & $77.2 \mathrm{a}$ & 177.2 & $267.8 \mathrm{a}$ & $0.3 \mathrm{~g}$ & $\ldots$ & $0.4 \mathrm{~d}$ & $\ldots$ \\
\hline Chlorothalonil 1,2,3,4 & $10.58 \mathrm{~b}$ & $71.4 \mathrm{a}$ & 179.4 & $261.4 \mathrm{a}$ & $0.8 \mathrm{fg}$ & $\ldots$ & $2.3 \mathrm{~d}$ & $\ldots$ \\
\hline Chlorothalonil 1,2 & $7.61 \mathrm{c}$ & $58.4 \mathrm{bc}$ & 182.2 & $248.2 \mathrm{~b}$ & $1.5 \mathrm{~d}-\mathrm{f}$ & $\ldots$ & $5.1 \mathrm{c}$ & $\ldots$ \\
\hline Chlorothalonil 2,3 & $8.04 \mathrm{c}$ & $63.2 \mathrm{~b}$ & 177.7 & $248.9 \mathrm{~b}$ & $1.0 \mathrm{e}-\mathrm{g}$ & $\ldots$ & $2.5 \mathrm{~d}$ & $\ldots$ \\
\hline Chlorothalonil 2 & $6.52 \mathrm{c}-\mathrm{e}$ & $58.2 \mathrm{bc}$ & 179.7 & $244.5 \mathrm{bc}$ & $2.2 \mathrm{~cd}$ & $\ldots$ & $5.1 \mathrm{c}$ & $\ldots$ \\
\hline Chlorothalonil 3,4 & $6.89 \mathrm{~cd}$ & $58.3 \mathrm{bc}$ & 170.4 & $235.6 \mathrm{~cd}$ & $4.1 \mathrm{~b}$ & $\ldots$ & $6.2 \mathrm{bc}$ & $\ldots$ \\
\hline Chlorothalonil 3 & $5.65 \mathrm{de}$ & $50.2 \mathrm{de}$ & 173.4 & $229.2 \mathrm{de}$ & $4.3 \mathrm{~b}$ & $\ldots$ & $8.0 \mathrm{~b}$ & $\ldots$ \\
\hline Propiconazole 1,2 & $6.54 \mathrm{c}-\mathrm{e}$ & $56.5 \mathrm{~b}-\mathrm{d}$ & 179.1 & $242.1 \mathrm{bc}$ & $1.9 \mathrm{c}-\mathrm{e}$ & $\ldots$ & $6.3 \mathrm{bc}$ & $\ldots$ \\
\hline Propiconazole 2 & $5.52 \mathrm{de}$ & $51.7 \mathrm{~cd}$ & 177.2 & $234.4 \mathrm{c}-\mathrm{e}$ & $2.6 \mathrm{c}$ & $\ldots$ & $7.1 \mathrm{bc}$ & $\ldots$ \\
\hline Untreated & $4.73 \mathrm{e}$ & $43.2 \mathrm{e}$ & 177.2 & $225.2 \mathrm{e}$ & $5.6 \mathrm{a}$ & $\ldots$ & $12.0 \mathrm{a}$ & $\ldots$ \\
\hline $\mathrm{CV}(\%)$ & 25.0 & 12.3 & 6.0 & 4.2 & 40.9 & $\ldots$ & 40.8 & $\ldots$ \\
\hline \multicolumn{9}{|l|}{ Field 92 E, Tama County, } \\
\hline \multicolumn{9}{|l|}{ Inbred 3} \\
\hline Mancozeb 1,2,3 & 11.68 & $69.1 \mathrm{a}$ & 112.8 & $193.5 \mathrm{a}$ & $\ldots$ & $\ldots$ & 3.7 & $39.0 \mathrm{c}$ \\
\hline Chlorothalonil 1,2,3 & 12.83 & $67.8 \mathrm{ab}$ & 106.8 & $187.4 \mathrm{ab}$ & $\ldots$ & $\ldots$ & 4.5 & $45.9 \mathrm{bc}$ \\
\hline Chlorothalonil 2,3 & 12.40 & $64.7 \mathrm{a}-\mathrm{c}$ & 115.8 & 192.9 a & $\ldots$ & $\ldots$ & 5.0 & $50.2 \mathrm{a}-\mathrm{c}$ \\
\hline Chlorothalonil 1,2 & 10.68 & $64.5 \mathrm{a}-\mathrm{c}$ & 119.1 & $194.3 \mathrm{a}$ & $\ldots$ & $\ldots$ & 8.4 & $48.7 \mathrm{bc}$ \\
\hline \multicolumn{9}{|l|}{ Propiconazole 1} \\
\hline + chlorothalonil 2 & 11.25 & $62.8 \mathrm{bc}$ & 118.0 & $192.0 \mathrm{a}$ & $\ldots$ & $\ldots$ & 3.9 & $56.4 \mathrm{ab}$ \\
\hline Propiconazole 1,2 & 10.60 & $62.3 \mathrm{bc}$ & 111.4 & $184.3 \mathrm{~b}$ & $\ldots$ & $\ldots$ & 5.2 & $55.9 \mathrm{ab}$ \\
\hline Chlorothalonil 1 & 9.80 & $61.7 \mathrm{c}$ & 118.0 & $189.5 \mathrm{ab}$ & $\ldots$ & $\ldots$ & 6.2 & $51.7 \mathrm{a}-\mathrm{c}$ \\
\hline Propiconazole 1 & 8.75 & $59.7 \mathrm{c}$ & 115.5 & $183.9 \mathrm{a}$ & $\ldots$ & $\ldots$ & 10.0 & $63.8 \mathrm{a}$ \\
\hline Chlorothalonil 2 & 10.38 & $59.2 \mathrm{c}$ & 113.3 & $182.8 \mathrm{~b}$ & $\ldots$ & $\ldots$ & 6.6 & $62.9 \mathrm{a}$ \\
\hline Untreated & 9.70 & $60.0 \mathrm{c}$ & 119.5 & $189.2 \mathrm{a}$ & $\ldots$ & $\ldots$ & 9.3 & $62.9 \mathrm{a}$ \\
\hline $\mathrm{CV}(\%)$ & 18.7 & 6.2 & 5.1 & 2.6 & $\ldots$ & $\ldots$ & 48.8 & 17.5 \\
\hline
\end{tabular}

${ }^{\mathrm{w}}$ Treatment means within columns followed by the same letter are not significantly different at $P=0.05$ according to the least significant difference test.

$\mathrm{x} \% \mathrm{LAD}=$ percentage of leaf area diseased: common rust (fields $92 \mathrm{~A}$ to $\mathrm{D}$ ); northern leaf spot (field $92 \mathrm{E}$ ). GS = growth stage.

${ }^{\mathrm{y}}$ For fields 92 A to D, $1=$ first spray, at GS $2.5 ; 2=$ second spray, at GS 4.0; $3=$ third spray, at GS 5.5; and $4=$ fourth spray, at GS 7.0. For field 92 E, $1=$ first spray, at GS 4.0; 2 = second spray, at GS 5.5; and 3 = third spray, at GS 7.0.

${ }^{\mathrm{z}}$ Coefficient of variation. 
$\left(\mathrm{SEE}_{\mathrm{y}}\right)$ were used to determine the assessment dates on which disease severity had the best relationship to corn seed yield $(12,16)$

\section{RESULTS}

1992. Moderate temperatures and moderately dry conditions in May and June preceded cool, wet weather during the reproductive phase of the seed corn crop (23). Common rust was the predominant disease in fields 92 A to D, whereas northern leaf spot was the predominant disease in field $92 \mathrm{E}$. Disease levels were lower in fields 92 A to D compared to field $92 \mathrm{E}$, but overall, untreated control plots yielded less than most fungicide-treated plots in all fields (Table 2). In field $92 \mathrm{~A}$, disease symptoms were not apparent by 16 August. Common rust developed after 16 August and a single disease assessment was made on 29 August at GS 8.2. Common rust severity (percentage of leaf area diseased [\%LAD]) was generally low (0.6 to $9.7 \%)$, but there were differences $(P \leq 0.05)$ among treatments, with the highest disease severity in untreated control plots and the lowest disease severity in plots treated with four sprays of mancozeb or four sprays of chlorothalonil. Large and medium seed units were greater from plots treated with four applications of mancozeb compared with other plots (Table 2).

In field $92 \mathrm{~B}$, the first detectable symptoms of common rust were observed on 28 July, 5 days before midsilk. At GS 7.0 and
8.5 , common rust severity was low ( 0.8 to $4.2 \%$ ), and differences in \% LAD at GS 8.5 among nine treatments, including the untreated control, were not significant $(P \leq$ $0.05)$. Only plots treated with four sprays of mancozeb had significantly lower common rust severity $(P \leq 0.05)$ at GS 8.5 compared with other treatments. There were significant differences among treatments $(P \leq 0.05)$ in the number of large, medium, and total salable seed units, with most treatments yielding more than the untreated control (Table 2).

In fields $92 \mathrm{C}$ and $\mathrm{D}$, very low, nonsignificant $(P \leq 0.05)$ levels of common rust severity were detected on 4 August. Subsequent disease assessments at GS 7.0 and GS 8.5 showed that common rust severity increased, with plots sprayed with mancozeb consistently having lower disease severity than unsprayed plots. Treatments in which fungicide sprays were initiated at GS 4.0 and continued through GS 5.5 or GS 7.0 had the lowest severity of common rust on the last assessment date at GS 8.5. On this date, common rust severity (\%LAD) ranged from $1 \%$ in plots treated with four sprays of mancozeb to $12 \%$ in untreated control plots, and differences $(P$ $\leq 0.05)$ in disease severity were observed among treatments (Table 2). Significant differences $(P \leq 0.05)$ in large, medium, and total salable seed units were observed among treatments, with plots treated with four sprays of mancozeb or four sprays of chlorothalonil yielding the greatest, and the
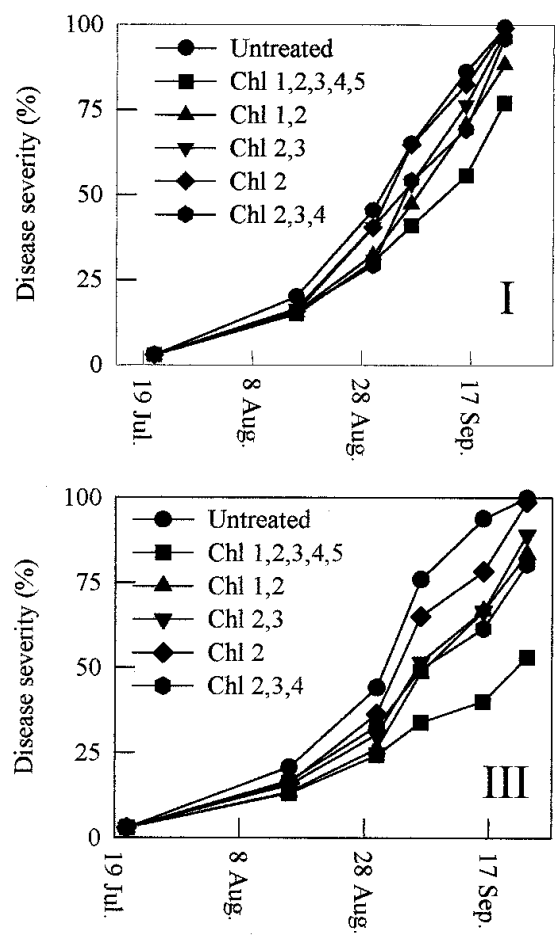

Disease assessment date
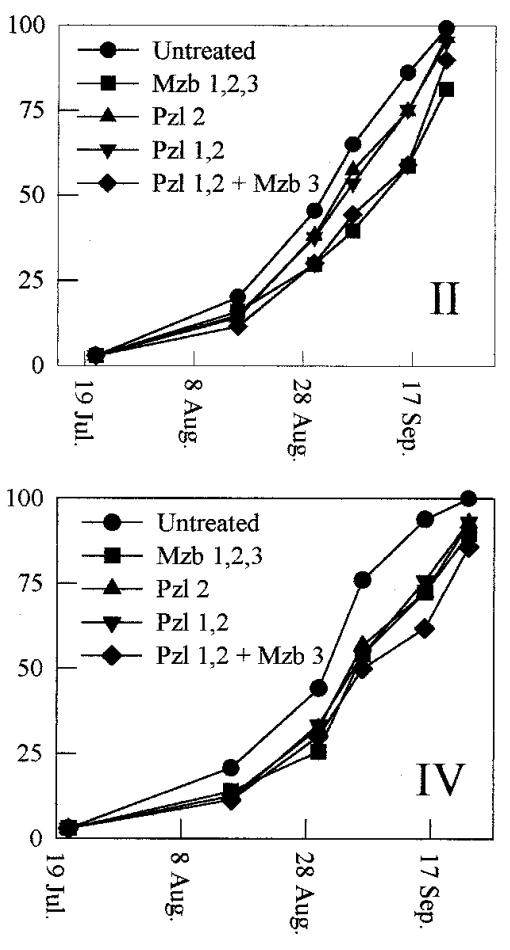

Disease assessment date

Fig. 1. Disease progress curves from fungicide experiments in hybrid corn seed fields $93 \mathrm{~A}$ (I and II) and $93 \mathrm{~B}$ (III and IV). Chl = chlorothalonil; Mzb = mancozeb; Pzl = propiconazole; 1,2,3,4,5 = first, second, third, fourth, and fifth sprays, respectively.

untreated control plots yielding the least number of salable seed units (Table 2).

The predominant disease in field $92 \mathrm{E}$ was northern leaf spot. The first symptoms of the disease were noticed on the last spray date, at GS 7.0. On this date, disease severity was very low, with a few scattered lesions limited to the third leaf below the ear leaf and lower leaves. A second disease severity assessment at GS 8.5 did not show significant differences $(P \leq 0.05)$ among treatments (Table 2). A final disease assessment on 16 September at GS 9.5 showed differences among treatments $(P \leq$ $0.05)$ with the lowest disease severity (\%LAD) in plots treated with three sprays of mancozeb or chlorothalonil (Table 2). Medium and total salable seed units differed $(P \leq 0.05)$ among treatments, whereas large and small units did not (Table 2).

1993. In 1993, there was heavy and persistent rainfall and temperatures were much cooler than normal (14) in all areas in which our experiments were conducted. The rainfall generally delayed planting. These conditions favored development of high levels of common rust severity. Disease severity increased from a few pustules per plant at the time plots were established to 1 to $4 \%$ of leaf area covered with lesions 2 weeks later, when a preliminary disease assessment was made on the first fungicide spray date.

Fields $93 \mathrm{~A}$ and $\mathrm{B}$, in which inbred 4 was planted, were severely affected by common rust. Whereas fungicide sprays somewhat slowed disease development in these fields, effective control was not achieved (Fig. 1) due to high common rust severity caused by the unusually cool, wet weather. Common rust severity (\%LAD) increased from about $3 \%$ on 21 July, when the first fungicide spray was applied, to about $7 \%$ on 30 July, approximately 1 week before detasseling. Differences in disease severity among plots were first observed on 24 August, when the fourth fungicide spray was applied. Plots with the lowest disease severity were those treated with three consecutive sprays of chlorothalonil, three consecutive sprays of mancozeb, or two propiconazole sprays on the first and second spray dates, followed by one spray of mancozeb on the third spray date (Table 3). In field $93 \mathrm{~A}$, significant differences $(P \leq 0.05)$ in standardized AUDPC, common rust severity on the fourth and fifth disease assessment dates, and the number of large, medium, small, and total seed units were obtained in response to different fungicide treatments (Table 3). In field $93 \mathrm{~B}$, differences among fungicide treatments in the number of small salable seed units were not significant at $P=0.05$ (Table 3). In field $93 \mathrm{~A}$, a $29 \%$ reduction in disease severity on the final assessment date in plots treated with five chlorothalonil sprays initiated on the first spray date $(\mathrm{Chl} 1,2,3,4,5)$ resulted in a 
Table 3. Large, medium, small, and total salable seed units, common rust severity on the last two assessment dates (4th and 5th AD), and standardized area under the disease progress curve (AUDPC) from fungicide treatments in experimental plots in hybrid corn seed production fields in Iowa in 1993

\begin{tabular}{|c|c|c|c|c|c|c|c|}
\hline Treatment $^{w}$ & $\begin{array}{c}\text { Large } \\
\text { units/ha }\end{array}$ & $\begin{array}{l}\text { Medium } \\
\text { units/ha }\end{array}$ & $\begin{array}{c}\text { Small } \\
\text { units/ha }\end{array}$ & $\begin{array}{c}\text { Total } \\
\text { units/ha }\end{array}$ & $\begin{array}{c}\text { \%LAD } \\
\text { (4th AD) }\end{array}$ & $\begin{array}{c}\text { \%LAD } \\
\text { (5th AD) }\end{array}$ & AUDPC \\
\hline \multicolumn{8}{|l|}{ Field 93 A, Benton County, Inbred 4} \\
\hline Chlorothalonil $1,2,3,4,5^{y}$ & $0.37 \mathrm{a}$ & $10.7 \mathrm{a}$ & $114.0 \mathrm{a}$ & $125.1 \mathrm{a}$ & $55.8 \mathrm{~d}$ & $77.2 \mathrm{c}$ & $46.0 \mathrm{e}$ \\
\hline Propiconazole $1,2+$ mancozeb 3 & $0.19 \mathrm{ab}$ & $6.8 \mathrm{bc}$ & $109.0 \mathrm{ab}$ & $116.0 \mathrm{ab}$ & $59.2 \mathrm{~d}$ & $90.0 \mathrm{~b}$ & $49.6 \mathrm{de}$ \\
\hline Mancozeb $1,2,3$ & $0.41 \mathrm{a}$ & $10.9 \mathrm{a}$ & $111.2 \mathrm{ab}$ & $122.5 \mathrm{a}$ & $58.7 \mathrm{~d}$ & $81.4 \mathrm{c}$ & $47.1 \mathrm{de}$ \\
\hline Chlorothalonil 1,2 & $0.37 \mathrm{a}$ & $7.4 \mathrm{ab}$ & $88.2 \mathrm{c}$ & $96.0 \mathrm{bc}$ & $70.8 \mathrm{c}$ & $88.5 \mathrm{~b}$ & $52.7 \mathrm{c}-\mathrm{e}$ \\
\hline Chlorothalonil $2,3,4$ & $0.13 \mathrm{ab}$ & $3.5 \mathrm{~cd}$ & $88.1 \mathrm{c}$ & $91.7 \mathrm{~b}-\mathrm{d}$ & $69.3 \mathrm{c}$ & $96.1 \mathrm{a}$ & $53.8 \mathrm{~cd}$ \\
\hline Chlorothalonil 2,3 & $0.05 \mathrm{~b}$ & $3.7 \mathrm{~b}-\mathrm{d}$ & $92.1 \mathrm{bc}$ & $95.9 \mathrm{bc}$ & $76.6 \mathrm{bc}$ & $97.8 \mathrm{a}$ & $59.0 \mathrm{bc}$ \\
\hline Propiconazole 1,2 & $0.05 \mathrm{~b}$ & $4.3 \mathrm{~b}-\mathrm{d}$ & $84.8 \mathrm{~cd}$ & $89.2 \mathrm{~cd}$ & $75.4 \mathrm{bc}$ & $95.4 \mathrm{a}$ & $56.8 \mathrm{bc}$ \\
\hline Propiconazole 2 & $0.08 \mathrm{~b}$ & $3.1 \mathrm{~cd}$ & $78.2 \mathrm{~cd}$ & $81.4 \mathrm{c}-\mathrm{e}$ & $75.0 \mathrm{bc}$ & $96.7 \mathrm{a}$ & $57.5 \mathrm{bc}$ \\
\hline Chlorothalonil 2 & $0.03 \mathrm{~b}$ & $1.5 \mathrm{~d}$ & $68.3 \mathrm{de}$ & $69.8 \mathrm{de}$ & $82.7 \mathrm{ab}$ & $99.1 \mathrm{a}$ & $62.4 \mathrm{ab}$ \\
\hline Untreated & $0.04 \mathrm{~b}$ & $1.5 \mathrm{~d}$ & $57.4 \mathrm{e}$ & $58.9 \mathrm{e}$ & $86.3 \mathrm{a}$ & $99.3 \mathrm{a}$ & $67.1 \mathrm{a}$ \\
\hline $\mathrm{CV}(\%)^{\mathrm{z}}$ & 122.2 & 78.4 & 25.0 & 27.0 & 19.5 & 8.7 & 9.7 \\
\hline \multicolumn{8}{|l|}{ Field 93 B, Benton County, Inbred 4} \\
\hline Chlorothalonil $1,2,3,4,5$ & $5.00 \mathrm{a}$ & $40.7 \mathrm{a}$ & 122.3 & $168.0 \mathrm{a}$ & $40.1 \mathrm{e}$ & $53.2 \mathrm{e}$ & $36.7 \mathrm{~d}$ \\
\hline Propiconazole $1,2+$ mancozeb 3 & $0.68 \mathrm{bc}$ & $19.3 \mathrm{bc}$ & 96.4 & $116.4 \mathrm{~b}$ & $62.0 \mathrm{~d}$ & $85.9 \mathrm{~b}-\mathrm{d}$ & $48.2 \mathrm{c}$ \\
\hline Mancozeb $1,2,3$ & $0.81 \mathrm{bc}$ & $17.9 \mathrm{~b}-\mathrm{d}$ & 104.5 & $123.2 \mathrm{~b}$ & $72.4 \mathrm{bc}$ & $80.6 \mathrm{~d}$ & $51.8 \mathrm{c}$ \\
\hline Chlorothalonil 1,2 & $2.02 \mathrm{~b}$ & $25.8 \mathrm{~b}$ & 94.3 & $122.1 \mathrm{~b}$ & $66.9 \mathrm{~cd}$ & $83.6 \mathrm{~cd}$ & $48.7 \mathrm{c}$ \\
\hline Chlorothalonil 2,3,4 & $1.50 \mathrm{bc}$ & $22.1 \mathrm{bc}$ & 98.8 & $122.4 \mathrm{~b}$ & $61.6 \mathrm{~d}$ & $89.8 \mathrm{bc}$ & $51.1 \mathrm{c}$ \\
\hline Chlorothalonil 2,3 & $0.76 \mathrm{bc}$ & $14.3 \mathrm{c}-\mathrm{e}$ & 103.1 & $118.2 \mathrm{~b}$ & $66.8 \mathrm{~cd}$ & $93.3 \mathrm{ab}$ & $52.2 \mathrm{c}$ \\
\hline Propiconazole 1,2 & $0.55 \mathrm{c}$ & $14.2 \mathrm{c}-\mathrm{e}$ & 98.1 & $112.9 \mathrm{~b}$ & $76.0 \mathrm{bc}$ & $89.3 \mathrm{~b}-\mathrm{d}$ & $53.7 \mathrm{c}$ \\
\hline Propiconazole 2 & $0.30 \mathrm{c}$ & $8.9 \mathrm{~d}-\mathrm{f}$ & 86.1 & $95.3 \mathrm{~b}$ & $72.5 \mathrm{bc}$ & $93.0 \mathrm{ab}$ & $53.8 \mathrm{c}$ \\
\hline Chlorothalonil 2 & $0.18 \mathrm{c}$ & 7.1 ef & 85.7 & $93.0 \mathrm{bc}$ & $78.5 \mathrm{~b}$ & $98.8 \mathrm{a}$ & $60.6 \mathrm{~b}$ \\
\hline Untreated & $0.13 \mathrm{c}$ & $3.9 \mathrm{f}$ & 66.3 & $70.3 \mathrm{c}$ & $93.9 \mathrm{a}$ & $100.0 \mathrm{a}$ & $71.1 \mathrm{a}$ \\
\hline $\mathrm{CV}(\%)$ & 130.5 & 67.1 & 24.9 & 28.2 & 18.8 & 15.9 & 7.6 \\
\hline \multicolumn{8}{|l|}{ Field 93 C, Marshall County, Inbred 5} \\
\hline Chlorothalonil $1,2,3,4,5$ & 1.81 & $15.7 \mathrm{a}-\mathrm{c}$ & 169.8 & 187.3 & $46.7 \mathrm{~g}$ & $63.3 \mathrm{c}$ & $27.0 \mathrm{e}$ \\
\hline Propiconazole $1,2+$ mancozeb 3 & 3.07 & $26.4 \mathrm{a}$ & 192.2 & 221.7 & $43.2 \mathrm{~g}$ & $82.2 \mathrm{~b}$ & $23.2 \mathrm{f}$ \\
\hline Mancozeb $1,2,3$ & 2.09 & $20.7 \mathrm{ab}$ & 171.7 & 194.5 & $57.6 \mathrm{ef}$ & $64.4 \mathrm{c}$ & $29.6 \mathrm{de}$ \\
\hline Chlorothalonil 1,2 & 1.64 & $14.2 \mathrm{bc}$ & 163.4 & 179.3 & $66.1 \mathrm{c}-\mathrm{e}$ & $75.1 \mathrm{bc}$ & $32.7 \mathrm{~cd}$ \\
\hline Chlorothalonil 2,3,4 & 1.82 & $12.9 \mathrm{c}$ & 159.4 & 174.1 & 64.9 de & $87.6 \mathrm{ab}$ & $35.9 \mathrm{~b}$ \\
\hline Chlorothalonil 2,3 & 1.67 & $18.2 \mathrm{a}-\mathrm{c}$ & 147.8 & 167.7 & $72.3 \mathrm{~b}-\mathrm{d}$ & $93.0 \mathrm{ab}$ & $35.5 \mathrm{bc}$ \\
\hline Propiconazole 1,2 & 1.25 & $18.0 \mathrm{a}-\mathrm{c}$ & 170.9 & 190.1 & $52.1 \mathrm{fg}$ & $87.5 \mathrm{ab}$ & $26.7 \mathrm{e}$ \\
\hline Propiconazole 2 & 0.79 & $11.4 \mathrm{c}$ & 165.2 & 177.4 & $74.9 \mathrm{a}-\mathrm{c}$ & $90.3 \mathrm{ab}$ & $36.5 \mathrm{~b}$ \\
\hline Chlorothalonil 2 & 1.31 & $12.1 \mathrm{c}$ & 152.8 & 166.2 & $77.3 \mathrm{ab}$ & $95.4 \mathrm{a}$ & $36.5 \mathrm{~b}$ \\
\hline Untreated & 1.48 & $12.1 \mathrm{c}$ & 141.2 & 154.8 & $82.4 \mathrm{a}$ & $94.9 \mathrm{a}$ & $42.7 \mathrm{a}$ \\
\hline $\mathrm{CV}(\%)$ & 59.1 & 34.2 & 14.3 & 12.7 & 15.0 & 17.0 & 6.9 \\
\hline \multicolumn{8}{|l|}{ Field 93 D, Grundy County, Inbred 5} \\
\hline Chlorothalonil $1,2,3,4,5$ & 0.34 & $4.3 \mathrm{ab}$ & $69.6 \mathrm{ab}$ & $74.2 \mathrm{ab}$ & $19.8 \mathrm{de}$ & $27.1 \mathrm{e}$ & $16.8 \mathrm{e}$ \\
\hline Propiconazole $1,2+$ mancozeb 3 & 0.34 & $4.3 \mathrm{ab}$ & $71.6 \mathrm{a}$ & $76.2 \mathrm{a}$ & $25.8 \mathrm{~cd}$ & $48.5 \mathrm{e}$ & $21.9 \mathrm{~d}$ \\
\hline Mancozeb $1,2,3$ & 0.45 & $5.1 \mathrm{a}$ & $67.6 \mathrm{a}-\mathrm{c}$ & $73.2 \mathrm{a}-\mathrm{c}$ & $13.9 \mathrm{e}$ & $34.3 \mathrm{e}$ & $14.1 \mathrm{e}$ \\
\hline Chlorothalonil 1,2 & 0.38 & $3.3 \mathrm{~b}-\mathrm{d}$ & $56.7 \mathrm{~cd}$ & $60.4 \mathrm{~cd}$ & $26.8 \mathrm{~cd}$ & $48.2 \mathrm{~d}$ & $21.6 \mathrm{~d}$ \\
\hline Chlorothalonil 2,3,4 & 0.22 & $2.7 \mathrm{c}-\mathrm{e}$ & $60.5 \mathrm{a}-\mathrm{d}$ & $63.4 \mathrm{a}-\mathrm{d}$ & $34.5 \mathrm{bc}$ & $59.5 \mathrm{bc}$ & $29.4 \mathrm{bc}$ \\
\hline Chlorothalonil 2,3 & 0.30 & $2.1 \mathrm{e}$ & $53.5 \mathrm{~d}$ & $55.9 \mathrm{~d}$ & $38.8 \mathrm{~b}$ & $65.9 \mathrm{~b}$ & $31.8 \mathrm{~b}$ \\
\hline Propiconazole 1,2 & 0.37 & $3.3 \mathrm{~b}-\mathrm{d}$ & $57.9 \mathrm{~b}-\mathrm{d}$ & $61.6 \mathrm{~b}-\mathrm{d}$ & $33.5 \mathrm{bc}$ & $52.1 \mathrm{~cd}$ & $25.7 \mathrm{~cd}$ \\
\hline Propiconazole 2 & 0.22 & $3.6 \mathrm{bc}$ & $56.8 \mathrm{~cd}$ & $60.6 \mathrm{~cd}$ & $39.9 \mathrm{~b}$ & $63.9 \mathrm{~b}$ & $30.8 \mathrm{~b}$ \\
\hline Chlorothalonil 2 & 0.36 & $2.5 \mathrm{de}$ & 59.0 a-d & $61.9 \mathrm{~b}-\mathrm{d}$ & $37.2 \mathrm{~b}$ & $66.8 \mathrm{~b}$ & $31.4 \mathrm{~b}$ \\
\hline Untreated & 0.11 & $1.7 \mathrm{e}$ & $48.2 \mathrm{~d}$ & $50.0 \mathrm{~d}$ & $60.0 \mathrm{a}$ & $85.1 \mathrm{a}$ & $45.0 \mathrm{a}$ \\
\hline $\mathrm{CV}(\%)$ & 61.7 & 39.1 & 17.7 & 18.0 & 15.1 & 31.4 & 10.3 \\
\hline \multicolumn{8}{|c|}{ Fields 93 E and F, Story County, Inbred 6} \\
\hline Chlorothalonil $1,2,3,4,5$ & $2.52 \mathrm{a}$ & 41.9 & 107.2 & 151.6 & $11.1 \mathrm{c}$ & $16.1 \mathrm{f}$ & $11.1 \mathrm{~d}$ \\
\hline Propiconazole $1,2+$ mancozeb 3 & $1.41 \mathrm{bc}$ & 28.9 & 105.9 & 136.2 & $10.7 \mathrm{c}$ & $22.2 \mathrm{e}$ & $11.6 \mathrm{~d}$ \\
\hline Mancozeb $1,2,3$ & $1.32 \mathrm{bc}$ & 31.7 & 106.0 & 139.0 & $10.7 \mathrm{c}$ & 19.8 ef & $11.3 \mathrm{~d}$ \\
\hline Chlorothalonil 1,2 & $2.00 \mathrm{ab}$ & 40.9 & 109.9 & 152.8 & $12.5 \mathrm{c}$ & $21.8 \mathrm{e}$ & $11.9 \mathrm{~d}$ \\
\hline Chlorothalonil 2,3,4 & $1.36 \mathrm{bc}$ & 35.8 & 114.9 & 152.1 & $16.0 \mathrm{~b}$ & $30.3 \mathrm{~d}$ & $15.5 \mathrm{bc}$ \\
\hline Chlorothalonil 2,3 & $1.59 \mathrm{a}-\mathrm{c}$ & 35.7 & 108.7 & 145.9 & $16.0 \mathrm{~b}$ & $32.7 \mathrm{~cd}$ & $15.8 \mathrm{~b}$ \\
\hline Propiconazole 1,2 & $0.93 \mathrm{c}$ & 27.7 & 110.9 & 139.5 & $14.3 \mathrm{bc}$ & $29.2 \mathrm{~d}$ & $13.7 \mathrm{c}$ \\
\hline Propiconazole 2 & $0.89 \mathrm{c}$ & 28.0 & 102.9 & 131.7 & $15.7 \mathrm{~b}$ & $37.5 \mathrm{bc}$ & $15.8 \mathrm{~b}$ \\
\hline Chlorothalonil 2 & $2.00 \mathrm{bc}$ & 36.9 & 111.9 & 150.7 & $17.0 \mathrm{~b}$ & $39.8 \mathrm{~b}$ & $17.3 \mathrm{~b}$ \\
\hline Untreated & $1.07 \mathrm{c}$ & 30.4 & 108.9 & 140.4 & $24.1 \mathrm{a}$ & $45.2 \mathrm{a}$ & $20.8 \mathrm{a}$ \\
\hline $\mathrm{CV}(\%)$ & 67.5 & 31.1 & 10.3 & 11.5 & 36.3 & 29.3 & 13.6 \\
\hline \multicolumn{8}{|c|}{ Fields $93 \mathrm{G}$ and $\mathrm{H}$, Webster County, Inbred 7} \\
\hline Chlorothalonil $1,2,3,4,5$ & 7.70 & $75.6 \mathrm{a}$ & 230.7 & $314.0 \mathrm{a}-\mathrm{c}$ & $11.2 \mathrm{~b}-\mathrm{d}$ & $28.7 \mathrm{~d}$ & $12.6 \mathrm{de}$ \\
\hline Propiconazole $1,2+$ mancozeb 3 & 7.31 & $80.3 \mathrm{a}$ & 241.9 & $329.6 \mathrm{a}$ & $10.6 \mathrm{~cd}$ & $28.0 \mathrm{~d}$ & $11.9 \mathrm{de}$ \\
\hline Mancozeb $1,2,3$ & 7.08 & $78.4 \mathrm{a}$ & 220.6 & $306.1 \mathrm{a}-\mathrm{d}$ & $9.1 \mathrm{~d}$ & $29.2 \mathrm{~d}$ & $11.3 \mathrm{e}$ \\
\hline Chlorothalonil 1,2 & 6.81 & $80.6 \mathrm{a}$ & 232.8 & $320.2 \mathrm{ab}$ & $10.7 \mathrm{~b}-\mathrm{d}$ & $32.9 \mathrm{~cd}$ & $12.9 \mathrm{c}-\mathrm{e}$ \\
\hline Chlorothalonil 2,3,4 & 5.74 & $66.9 \mathrm{ab}$ & 221.8 & 294.5 b-e & $14.6 \mathrm{a}$ & $34.6 \mathrm{~cd}$ & $13.4 \mathrm{~b}-\mathrm{e}$ \\
\hline Chlorothalonil 2,3 & 6.05 & $67.4 \mathrm{ab}$ & 214.6 & $288.0 \mathrm{c}-\mathrm{e}$ & $12.6 \mathrm{a}-\mathrm{c}$ & $37.7 \mathrm{bc}$ & $15.0 \mathrm{~b}$ \\
\hline Propiconazole 1,2 & 4.94 & $65.2 \mathrm{ab}$ & 214.6 & $284.7 \mathrm{c}-\mathrm{e}$ & $11.2 \mathrm{~b}-\mathrm{d}$ & $41.4 \mathrm{ab}$ & $13.5 \mathrm{~b}-\mathrm{d}$ \\
\hline Propiconazole 2 & 5.75 & $67.4 \mathrm{ab}$ & 225.1 & 298.3 b-e & $14.5 \mathrm{a}$ & $35.9 \mathrm{bc}$ & $14.8 \mathrm{bc}$ \\
\hline Chlorothalonil 2 & 4.68 & $65.0 \mathrm{ab}$ & 212.8 & $282.4 \mathrm{de}$ & $12.6 \mathrm{a}-\mathrm{c}$ & $35.0 \mathrm{~b}-\mathrm{d}$ & $15.1 \mathrm{bc}$ \\
\hline Untreated & 4.12 & $56.5 \mathrm{~b}$ & 212.7 & $273.3 \mathrm{e}$ & $13.9 \mathrm{ab}$ & $44.2 \mathrm{a}$ & $17.2 \mathrm{a}$ \\
\hline $\mathrm{CV}(\%)$ & 45.3 & 22.4 & 11.1 & 10.4 & 28.6 & 30.1 & 11.8 \\
\hline
\end{tabular}

${ }^{\mathrm{w}}$ Treatment means within columns followed by the same letter are not significantly different at $P=0.05$ according to the least significant difference test.

$x$ Percentage of leaf area diseased.

y 1 = first spray, 7 to 13 days before second spray; 2 = second spray, 1 to 4 days before first day of detasseling; $3=$ third spray, 1 to 4 days after last day of detasseling; 4 = fourth spray, 10 to 13 days after third spray; and 5 = fifth spray, 10 to 11 days after fourth spray.

${ }^{\mathrm{z}}$ Coefficient of variation. 

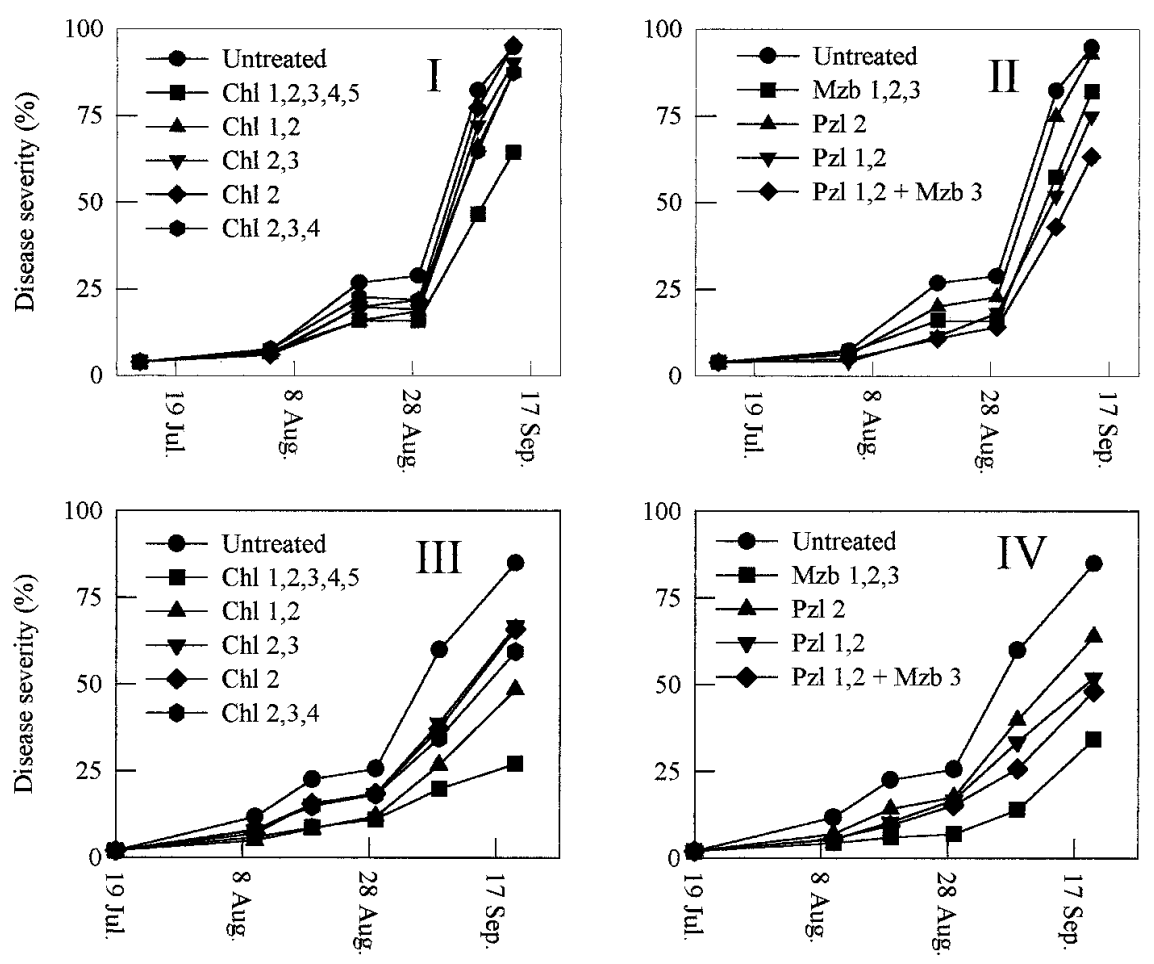

Disease assessment date

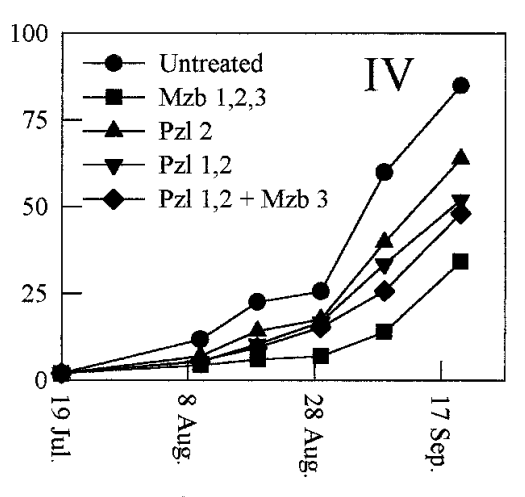

Disease assessment date

Fig. 2. Disease progress curves from fungicide experiments in hybrid corn seed fields $93 \mathrm{C}$ (I and II) and $93 \mathrm{D}$ (III and IV). Chl = chlorothalonil; $\mathrm{Mzb}=$ mancozeb; Pzl = propiconazole; 1,2,3,4,5 = first, second, third, fourth, and fifth sprays, respectively.
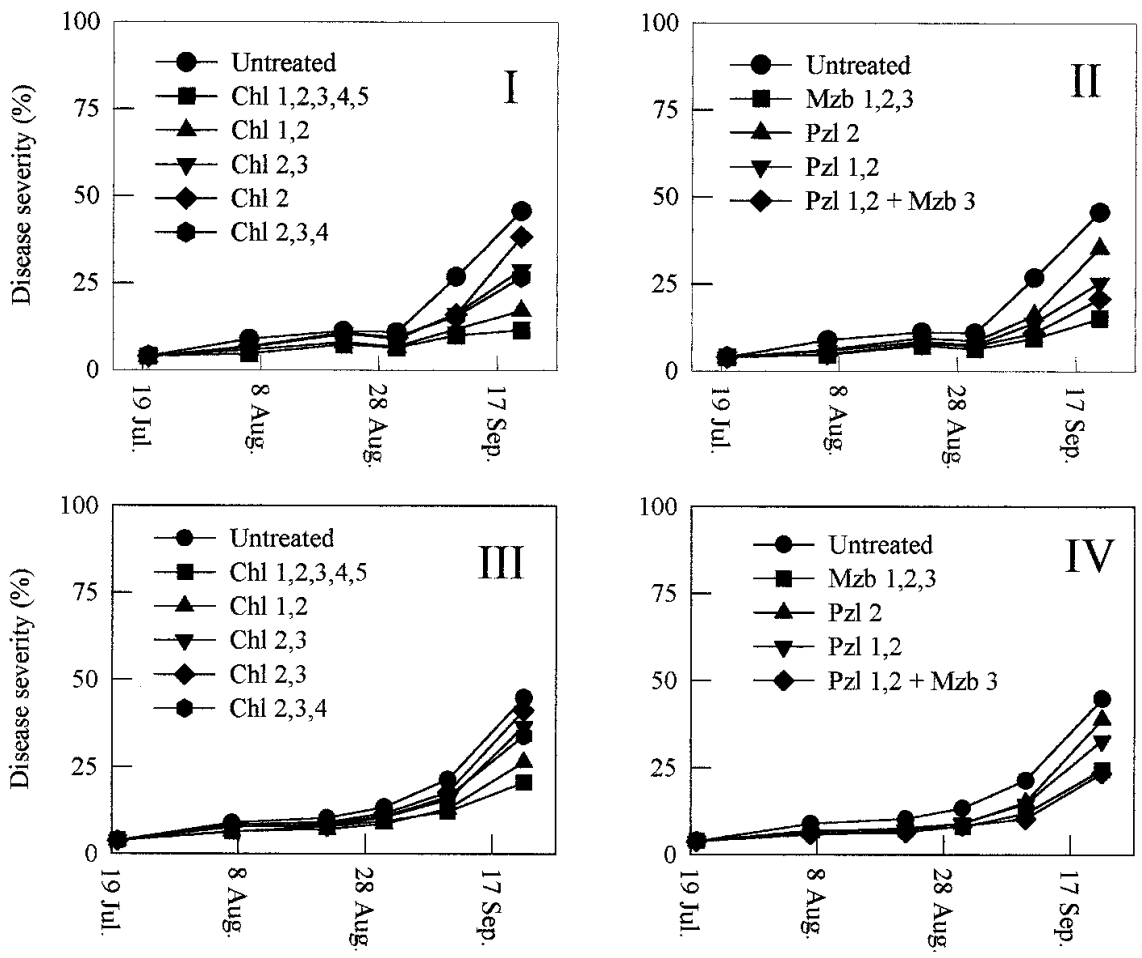

Disease assessment date

Disease assessment date

Fig. 3. Disease progress curves from fungicide experiments in hybrid corn seed fields 93 E (I and II) and $93 \mathrm{~F}$ (III and IV). Chl = chlorothalonil; Mzb = mancozeb; Pzl = propiconazole; 1,2,3,4,5 = first, second, third, fourth, and fifth sprays, respectively.

$112 \%$ increase in total units of salable seed. In field $93 \mathrm{~B}$, the same treatment reduced disease severity on the final assessment date by $89 \%$ and increased total units of salable seed by $140 \%$.

In field $93 \mathrm{C}$, percentage of leaf area covered with common rust was approximately $4 \%$ when fungicide sprays were initiated on 13 July. Visually discernible differences among fungicide-treated plots were first observed on 19 August, 8 days after the third fungicide spray was applied. On this date, plots with the lowest common rust severity were those treated with two sprays of propiconazole initiated on the first spray date, followed by one mancozeb spray on the third spray date (Pzl 1,2+ Mzb 3). By 14 September, when final disease severity was assessed, this treatment and Chl 1,2,3,4,5 had the lowest common rust severity (Table 3 ). Differences among treatments in medium units of salable seed were significant $(P \leq 0.05$; Table 3$)$. Pzl 1 , $2+$ Mzb 3 reduced disease severity on the final assessment date by $51 \%$ and increased total units of salable seed by $43 \%$.

Percentage of leaf area covered with common rust in field $93 \mathrm{D}$ was approximately $2 \%$ on 19 July, when a preliminary disease assessment was made. Standardized AUDPC and severity of common rust on the fourth and fifth disease assessment dates were higher in untreated control plots $(P \leq 0.05)$ than in most fungicide-treated plots (Table 3 ). The most effective disease control was achieved with Chl 1,2,3,4,5 and three consecutive mancozeb sprays initiated on the first spray date (Mzb 1,2,3; Fig. 2). There were significant differences $(P \leq 0.05)$ among treatments in medium, small, and total salable seed units (Table 3). Chl 1,2,3,4,5 reduced disease severity on the final assessment date by $215 \%$ and increased total units of salable seed by $48 \%$.

In fields $93 \mathrm{E}$ and $\mathrm{F}$, common rust severity was $4 \%$ on the preliminary assessment date (20 July). Disease severity was generally low during the growing season (Fig. 3), was lowest on the final assessment date in plots treated with $\mathrm{Chl}$ $1,2,3,4,5$, and was highest in untreated control plots (Table 3 ). Differences among treatments in standardized AUDPC and common rust severity on the fourth and fifth disease assessment dates were significant $(P \leq 0.05$; Table 3$)$. Plots treated with Chl 1,2,3,4,5 yielded the most units of salable seed (Table 3 ).

In fields $93 \mathrm{G}$ and $\mathrm{H}$, severity of common rust in untreated control plots increased from $2 \%$ on 19 July to 34 and $54 \%$, respectively, on the final assessment date, 17 September (Fig. 4). There were differences $(P \leq 0.05)$ in standardized AUDPC and common rust severity on the fourth and fifth disease assessment dates (Table 3). Total units of salable seed differed among treatments $(P \leq 0.05)$. Plots treated with Chl 1,2,3,4,5, Mzb 1,2,3, or 
Pzl 1,2 + Mzb 3 had higher yields than other plots, whereas unsprayed control plots had lower yields than all fungicidetreated plots (Table 3).

Critical point models. In field $92 \mathrm{~A}$, a single assessment of common rust severity at GS 8.2 explained $74 \%$ of the variation in corn seed yield (Table 4). In fields 92 B-D, critical point models using common rust severity assessments at GS 8.5 explained more of the variation in corn seed yield than critical point models using disease severity assessments at GS 7.0 (Table 4). In field $92 \mathrm{E}$, northern leaf spot severity assessed at GS 9.5 explained $42 \%$ of the variation in corn seed yield, compared to $8 \%$ of the variation in yield when disease severity was assessed at GS 8.5 (Table 4).

In fields $93 \mathrm{~A}$ to $\mathrm{D}$, critical point models using common rust severity assessments on the fourth date explained more of the variation in corn seed yield and had lower $\mathrm{SEE}_{\mathrm{y}}$ than critical point models using common rust severity assessed on the fifth date (Table 4). In fields $93 \mathrm{E}$ to $\mathrm{H}$, common rust severity assessed on the fifth date explained more of the variation in corn seed yield and had lower $\mathrm{SEE}_{\mathrm{y}}$ than common rust severity assessed on the fourth date (Table 4). In 1993, critical point models using AUDPC explained nearly as much of the variation in corn seed yield as the best critical point models using common rust severity on a single assessment date.

\section{DISCUSSION}

The exceptionally wet and cool conditions in 1993 compared to 1992 favored higher common rust severities in 1993. Common rust epidemics in 1993 were compounded by late planting caused by heavy and persistent rainfall. Consequently, damaging levels of common rust affected the seed corn crop at the most critical stages of development, that is, before tasseling through about 4 weeks after tasseling (4). Differences in disease severity between fungicide-treated and untreated plots were greater in 1993 compared to 1992. Within years, yield differences were greater in experiments with high disease severities compared to experiments with low disease severities. Therefore, fungicide applications provided the greatest increase in yield when disease severity levels were high. These results indicate that when conditions are favorable for development of common rust (cool, wet weather), foliar applications of chlorothalonil, mancozeb, or propiconazole may minimize yield losses due to foliar diseases in hybrid corn seed production.

The yield components most responsible for the reduction in salable seed yield as affected by foliar pathogens of corn are number and kernel size $(1,4,34)$. This was demonstrated in this study, in which fungicide applications for control of common rust increased the number of salable seed
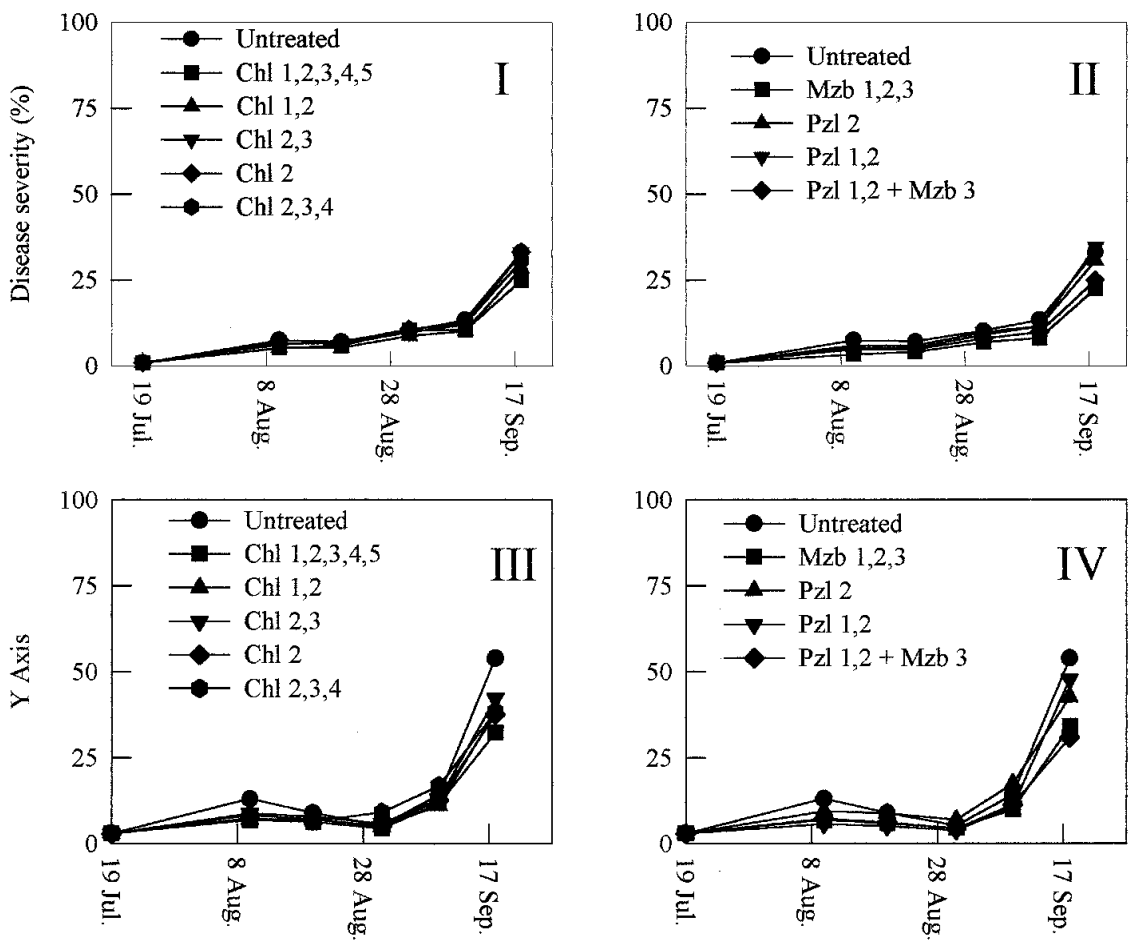

Disease assessment date

Disease assessment date

Fig. 4. Disease progress curves from fungicide experiments in hybrid corn seed fields 93 G (I and II) and $93 \mathrm{H}$ (III and IV). Chl = chlorothalonil; Mzb = mancozeb; Pzl = propiconazole; 1,2,3,4,5 = first, second, third, fourth, and fifth sprays, respectively.

Table 4. Coefficients of determination $\left(\mathrm{R}^{2}\right)$, intercepts, slopes, and standard errors of the Y-estimate $\left(\mathrm{SEE}_{\mathrm{y}}\right)$ from regressions of yield (total units/ha) on disease severity assessed at two growth stages (last two assessments); and on standardized area under the disease progress curve (AUDPC) in fungicide trial experiments in commercial hybrid corn seed production fields in Iowa in 1992 and 1993

\begin{tabular}{|c|c|c|c|c|c|}
\hline Field/s & GSv/ADw/AUDPC & Intercept & Slope $^{\mathrm{x}}$ & $\mathbf{R}^{2}$ & $\operatorname{SEE}^{\mathbf{y}}$ \\
\hline \multirow[t]{2}{*}{$92 \mathrm{~A}$} & 7.0 & $\ldots$ & $\ldots$ & $\ldots$ & $\ldots$ \\
\hline & 8.2 & 220 & $-1.6 * *$ & 0.74 & 2.0 \\
\hline \multirow[t]{2}{*}{$92 \mathrm{~B}$} & 7.0 & 205 & -2.4 & 0.03 & 13.0 \\
\hline & 8.5 & 225 & -8.0 & 0.30 & 14.3 \\
\hline \multirow[t]{2}{*}{$92 \mathrm{C}$ and $\mathrm{D}$} & 7.0 & 244 & $-7.2 * *$ & 0.83 & 3.3 \\
\hline & 8.5 & 265 & $-3.8 * *$ & 0.88 & 3.2 \\
\hline \multirow[t]{2}{*}{$92 \mathrm{E}$} & 8.5 & 192 & -0.5 & 0.08 & 4.2 \\
\hline & 9.5 & 207 & $-0.3 *$ & 0.43 & 7.5 \\
\hline \multirow[t]{3}{*}{$93 \mathrm{~A}$} & IV $^{y}$ & 240 & $-2.0 * *$ & 0.93 & 13.7 \\
\hline & $\mathrm{V}^{\mathrm{z}}$ & 322 & $-2.5 * *$ & 0.76 & 45.2 \\
\hline & AUDPC & 267 & $-3.1 * *$ & 0.92 & 18.0 \\
\hline \multirow[t]{3}{*}{$93 \mathrm{~B}$} & IV & 234 & $-1.7 * *$ & 0.88 & 15.7 \\
\hline & V & 267 & $-1.8 * *$ & 0.84 & 24.0 \\
\hline & AUDPC & 259 & $-2.8 * *$ & 0.90 & 17.2 \\
\hline \multirow[t]{3}{*}{$93 \mathrm{C}$} & IV & 260 & $-1.2 * *$ & 0.78 & 14.9 \\
\hline & V & 249 & -0.8 & 0.27 & 39.7 \\
\hline & AUDPC & 276 & $-2.9 * *$ & 0.84 & 14.7 \\
\hline \multirow[t]{3}{*}{$93 \mathrm{D}$} & IV & 82 & $-0.6 * *$ & 0.75 & 4.1 \\
\hline & V & 87 & $-0.4 * *$ & 0.73 & 5.2 \\
\hline & AUDPC & 85 & $-0.8 * *$ & 0.73 & 4.8 \\
\hline \multirow[t]{3}{*}{$93 \mathrm{E}$ and $\mathrm{F}$} & IV & 146 & -0.1 & 0.00 & 10.2 \\
\hline & V & 149 & -0.2 & 0.05 & 8.6 \\
\hline & AUDPC & 147 & -0.2 & 0.01 & 12.6 \\
\hline \multirow[t]{3}{*}{$93 \mathrm{G}$ and $\mathrm{H}$} & IV & 368 & -5.6 & 0.33 & 34.6 \\
\hline & V & 400 & $-2.9 * *$ & 0.73 & 21.6 \\
\hline & AUDPC & 413 & $-8.3 * *$ & 0.64 & 30.0 \\
\hline
\end{tabular}

v Growth stage.

${ }^{\mathrm{w}}$ Disease severity assessment date.

$\mathrm{x} *=$ Slope significantly different from 0 at $P \leq 0.05$ and $* *=$ slope significantly different from 0 at $P \leq 0.01$.

y Fourth disease severity assessment date, 4 to 5 weeks after 3 to $6 \%$ seed parent silking.

${ }^{\mathrm{z}}$ Fifth disease severity assessment date, 6 to 7 weeks after 3 to $6 \%$ seed parent silking. 
units within the three seed size categories (large, medium, or small).

Because foliar diseases of seed corn are most damaging when infections occur during the crop period that begins before tasseling and continues until approximately 4 weeks after tasseling (4), timing of fungicide sprays is important. Our results suggest that the best disease control can be achieved when a fungicide spray program starts early (at low disease severity levels) and sprays are continued for at least three consecutive application dates.

At least one or two sprays should be applied before female inbred silking, or sprays should be started soon after disease detection. Our results are similar to those of Pataky (18), who found that there was an inverse relationship between the number of mancozeb or propiconazole applications and common rust severity on hybrid sweet corn. Pataky also reported that five fungicide applications resulted in the lowest disease severity levels. Ward et al. (32), found that the most effective control of gray leaf spot (Cercospora zeae-maydis Tehon and Daniels) as well as the highest grain yields were achieved when fungicide sprays were initiated at disease severity levels of 2 to $3 \%$ with applications continuing until physiological maturity. The studies by Pataky, Ward et al., and ours demonstrate the importance of fungicide spray timing to achieve maximum disease control and yield benefits in corn. In our study, 3 to 5 sprays of chlorothalonil, mancozeb, or a combination of propiconazole and mancozeb resulted in lower disease severities and higher yields than zero, one, or two applications of the same fungicides.

Common rust severity was relatively low in 1992 in fields $92 \mathrm{~A}$ to D and in 1993 in fields $93 \mathrm{E}$ to $\mathrm{H}$ compared to fields $92 \mathrm{E}$ and 93 A to D, yet large and medium salable seed units increased in response to fungicide applications. This yield increase in the presence of low common rust severity levels may have been due, in part, to a phytotonic effect of fungicides. This stimulatory effect of fungicide treatments on growth may result in significant yield increases even in the absence of disease (7) and has been demonstrated in spring barley $(7,31)$ and in coffee $(30)$. In the presence of low levels of disease severity, as in this study, fungicide treatments may, if applied at critical stages of development, still increase yield by reducing or eliminating the negative effects of other non-target pathogens, delaying senescence, or directly affecting plant growth (7).

Information provided in this study on the efficacy of fungicide treatments for control of common rust and northern leaf spot in corn inbreds, as well as information on the economic benefits of using fungicides to control foliar pathogens of corn inbreds (34), may be useful to seed companies and growers by helping them to make improved pest management decisions regarding the use of fungicides. More research is required to identify high-risk areas for corn disease epidemics and to develop disease-forecasting systems in hybrid corn seed production.

\section{ACKNOWLEDGMENTS}

We thank D. Cox, Department of Statistics, Iowa State University, for advice on statistical analysis; C. Fish and T. Kemper, Pioneer Hi-Bred International, Toledo, Iowa, W. Houser, Pioneer Hi-Bred International, Marengo, Iowa, D. Hoffman, ICI Seeds, Coon Rapids, Iowa, and T. Primus, Lynks Seeds Inc., Marshalltown, Iowa for their cooperation; and G. Tylka and G. Munkvold, Department of Plant Pathology, Iowa State University, and $\mathrm{H}$. Scherm, Department of Plant Pathology, University of Georgia (formerly Iowa State University) for critical reading of the manuscript.

\section{LITERATURE CITED}

1. Bowen, K. L., and Pedersen, W. L. 1988. Effects of northern leaf blight and detasseling on yields and yield components of corn inbreds. Plant Dis. 72:952-956.

2. Campbell, C. L., and Madden, L. V. 1990. Introduction to Plant Disease Epidemiology. John Wiley \& Sons, New York.

3. Dillard, H. R., and Seem, R. C. 1990. Use of an action threshold for common maize rust to reduce crop loss in sweet corn. Phytopathology 80:846-849.

4. Edwards, C. R. 1992. Seed Corn Pest Management Manual for the Midwest. Purdue Univ. Coop. Ext. Serv. Dep. Bot. Plant Pathol. Entomol.

5. Fisher, D. E., Hooker, A. L., Lim, S. M., and Smith, D. R. 1976. Leaf infection and yield loss caused by four Helminthosporium leaf diseases of corn. Phytopathology 66:942-944.

6. Fry, W. E. 1978. Quantification of general resistance of potato cultivars and fungicide effects for integrated control of potato late blight. Phytopathology 68:1650-1655.

7. Griffiths, E., and Scott, S. W. 1977. Possible phytotonic effects of fungicides on barley. Pages 465-477 in: Crop Protection Agents: Their Biological Evaluation. Proc. Int. Conf. on the Evaluation of Biological Activity. N. R. McFarlane, ed. Academic Press, London.

8. Groth, J. V., Zeyen, R. L., Davis, D. W., and Christ, B. J. 1983. Yield and quality losses caused by common rust (Puccinia sorghi Schw.) in sweet corn (Zea Mays) hybrids. Crop Prot. 2:105-111.

9. Hanway, J. J. 1963. Growth stages of corn (Zea mays L.). Agron. J. 55:487-491.

10. Hunter, R. B., Mortimore, C. G., and Kannenberg, L. W. 1973. Inbred maize performance following tassel and leaf removal. Agron. J. 65:471-472.

11. James, C. 1971. A Manual of Assessment Keys for Plant Diseases. Can. Dep. Agric. Publ. No. 1458.

12. Madden, L. V., and Nutter, F. W., Jr. 1995. Modeling crop losses at the field scale. Can. J. Plant Pathol. 17:124-137.

13. Martinson, C. A., Wegulo, S. N., Rivera-C, J. M., and Nutter, F. W., Jr. 1994. Fungicidal spray program for seed corn production. Pages 71-79 in: Proc. Annu. Corn Sorghum Res. Conf., 49th. D. Wilkinson, ed. American Seed Trade Association, Washington, DC.

14. Munkvold, G. P., and Yang, X. B. 1995. Crop damage and epidemics associated with 1993 floods in Iowa. Plant Dis. 79:95-101.

15. Newhouse, K. 1994. Detasselor - A new solution for the 'Detassel Hassle.' Pages 97-103 in: Proc. Annu. Corn Sorghum Res. Conf., 49th. D. Wilkinson, ed. American Seed Trade Association, Washington, DC.

16. Nutter, F. W., Jr., and Littrell, R. H. 1996. Relationships among defoliation, canopy reflectance, and pod yield in the peanut-late leafspot pathosystem. Crop Prot.15:135-142.

17. Nutter, F. W., Jr., and Parker, S. K. 1997. Fitting disease progress curves using EPIMODEL. Pages 24-28 in: Exercises in Plant Disease Epidemiology. L. Francl and D. Neher, eds. APS Press, St. Paul, MN.

18. Pataky, J. K. 1987. Quantitative relationships between sweet corn yield and common rust, Puccinia sorghi. Phytopathology 77:10661071.

19. Pataky, J. K., and Eastburn, D. M. 1993. Comparing partial resistance to Puccinia sorghi and applications of fungicides for controlling common rust on sweet corn. Phytopathology 83:1046-1051.

20. Pataky, J. K., and Headrick, J. M. 1989. Management of common rust on sweet corn with resistance and fungicides. J. Prod. Agric. 2:362-369.

21. Paulus, A. O., Nelson, J., Otto, H. W., and Hall, B. J. 1986. Comparison of fungicides for control of sweet corn rust. Calif. Agric. 40:5-6.

22. Petersen, R. G. 1994. Agricultural Field Experiments: Design and Analysis. M. Dekker, New York.

23. Rivera-Canales, J. M. 1993. Yield losses, chemical control, and epidemiology of fungal leaf blights on seed corn in Iowa. (Abstr.) Ph.D. Diss. Microfilm Order No. ISU 1993 R525, Iowa State University, Ames.

24. Shaner, G., and Finney, R. E., 1977. The effect of nitrogen fertilization on the expression of slow-mildewing resistance in Knox wheat. Phytopathology 67:1051-1056.

25. Smith, D. R., and White, D. G. 1988. Diseases of corn. Pages 687-766 in: Corn and Corn Improvement, 3rd ed. American Society of Agronomy. Madison, WI.

26. Steel, R. G. D., Torrie, J. H., and Dickey, D. A. 1997. Principles and Procedures of Statistics: A Biometrical Approach. McGraw-Hill, New York.

27. Sumner, D. R., Doupnik, B., Jr., and Boosalis, M. G. 1981. Effects of reduced tillage and multiple cropping on plant diseases. Annu. Rev. Phytopathol. 19:167-187.

28. Ullstrup, A. J., and Miles, S. R. 1957. The effects of some leaf blights of corn on grain yield. Phytopathology 47:331-336.

29. Vasilas, B. L., and Seif, R. D. 1985. Pre-anthesis defoliation effects on six corn inbreds. Agron. J. 77:831-835.

30. Vossen, H. A. M. van der. 1982. Consequences of phytotonic effects of fungicide to breeding for disease resistance, yield and quality in Coffea arabica L. J. Hortic. Sci. 57:321-329.

31. Wale, S. J., and Hunter, E. A. 1985. A comparison of fungicides for the control of mildew on spring barley. J. Agric. Sci. 105:573-579.

32. Ward, J. M. J., Laing, M. D., and Rijkenberg, F. H. J. 1997. Frequency and timing of fungicide applications for the control of gray leaf spot in maize. Plant Dis. 81:41-48.

33. Wegulo, S. N. 1994. Benefits assessment of fungicide usage in seed corn production in Iowa. M. S. thesis. Iowa State University, Ames.

34. Wegulo, S. N., Martinson, C. A., Rivera-C, J. M., and Nutter, F. W., Jr. 1996. Model for economic analysis of fungicide usage in hybrid corn seed production. Plant Dis. 81:415-422.

35. Wych, R. D. 1988. Production of hybrid seed corn. Pages 565-607 in: Corn and Corn Improvement, 3rd ed. American Society of Agronomy. Madison, WI. 